\title{
Organic Rankine cycle power plant by low-temperature slag washing water of blast furnace
}

\author{
Xiaoyu Chen $^{1 \mathrm{a}}$, Yanna Liu ${ }^{2 b}$, Song Xiao ${ }^{1 \mathrm{c}, \dagger}$ \\ ${ }^{1}$ Faculty of Methallurgical and Energy Engineering, Kunming University of Science and Technology, \\ Kunming, 650093, China \\ ${ }^{2}$ Faculty of Chemical Engineering, Kunming University of Science and Technology, Kunming, \\ 650093, China \\ a690569390@qq.com, bnuo2004lyn@163.com, xxskmust@163.com
}

\begin{abstract}
Keywords: Slag-washing water, Organic Rankine cycle, Power plant, Low temperature.
Abstract. Three typical organic fluids for Organic Rankine power cycles utilizing low-temperature slag washing water of blast furnace is presented. With the aim of making the best use of the energy contained in a stream of $60-80^{\circ} \mathrm{C}$ slag washing water of blast furnace. The available heat flux of R125 is the highest, and the value of the minimum can reach $70 \%$. When the temperature of initial slag washing water temperature is lower than $70^{\circ} \mathrm{C}$, the work fluids $\mathrm{R} 125$ and R245fa have the equal theoretical power and higher than the work fluid R143a.
\end{abstract}

\section{Introduction}

Due to the increasingly severe energy crisis and environment pollution, the waste heat recovery of industries is significant as it can reduce energy consumption and reduce $\mathrm{CO}_{2}$ emissions. Organic Rankine cycle (ORC) is a promising solution because of its flexibility, safety, low maintenance requirements and outstanding economical performance $[1,2]$.

Compare with the conventional steam Rankine cycle, ORC can get relatively high efficiency for the conversion of low-grade heat into power [3-5]. The choice of working fluids is of key importance for the performance of an ORC. For example, HCFC123, HFC-245fa, HFC-245ca, isobutene are as organic fluids have been studied for use in ORC systems [6-8].

In this paper, three typical organic fluids for organic Rankine power cycles utilizing low-temperature slag washing water of blast furnace is presented. In this system, the thermal efficiency, efficiency of utilization of slag washing water of blast furnace, power and temperature of injected slag washing water of blast furnace will be analyzed by different temperature of slag washing water of blast furnace. The results can help us to choose the suitable organic fluids for organic Rankine power cycles.

\section{Model and assumption}

In this section, the schematic of the ORC and T-S diagram of the cycle are shown in Figs. 1 and 2, respectively. The present ORC system consists of heat exchangers (preheater and vaporizer), a vapor turbine, a condenser and a pump. For all work fluids the starting point for the energy analysis is the assumption that slag washing water is available at temperature $T_{S W W 1}\left(60-80^{\circ} \mathrm{C}\right)$ and at a constant flow rate of $30 \mathrm{~kg} / \mathrm{s}$. It is assumed that the condensing temperature of the work fluid and the pinch point in the slag washing water heat exchange process are $20^{\circ} \mathrm{C}$ and $5^{\circ} \mathrm{C}$, respectively.

When the rejection temperature for slag washing water is equal into $30^{\circ} \mathrm{C}$ (i.e., $T_{S W W 2}^{\min }=30^{\circ} \mathrm{C}$ ), the greatest utilization of slag washing water energy will be obtained. The slag washing water heat flux available for utilization can be defined as: 


$$
Q_{S W W}=m_{S W W} C_{P-S W W}\left(T_{S W W 1}-T_{S W W 2}{ }^{\min }\right)
$$

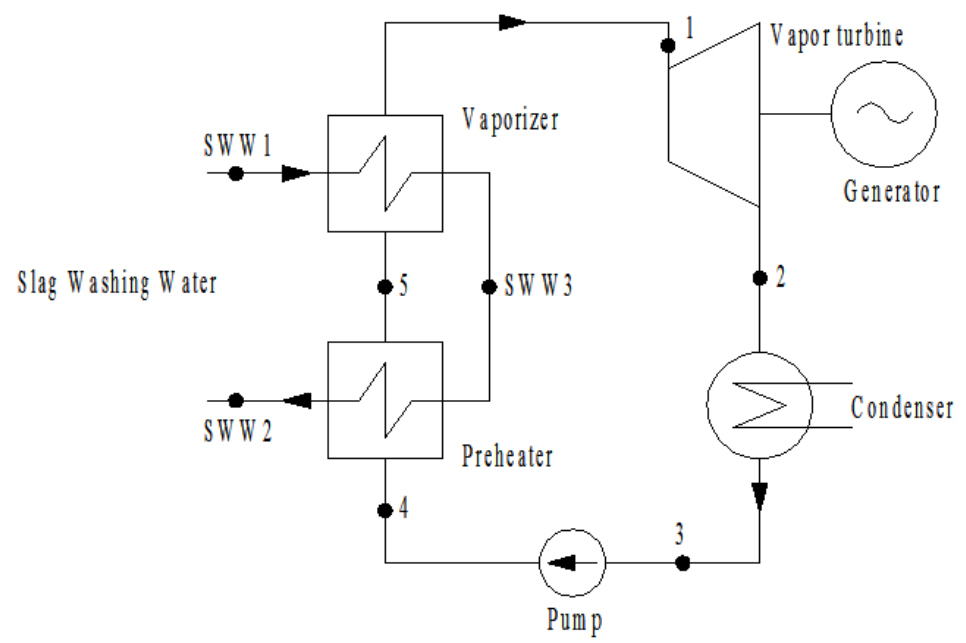

Fig. 1. Schematic of an organic Rankine cycle power plant

Where $m_{S W W}$ is the slag washing water mass flow rate $(\mathrm{kg} / \mathrm{s}), C_{P-S W W}$ is the specific heat of slag washing water $(\mathrm{kJ} / \mathrm{kg} \mathrm{K}), T_{S W W 1}$ is the slag washing water temperature $\left({ }^{\circ} \mathrm{C}\right)$.

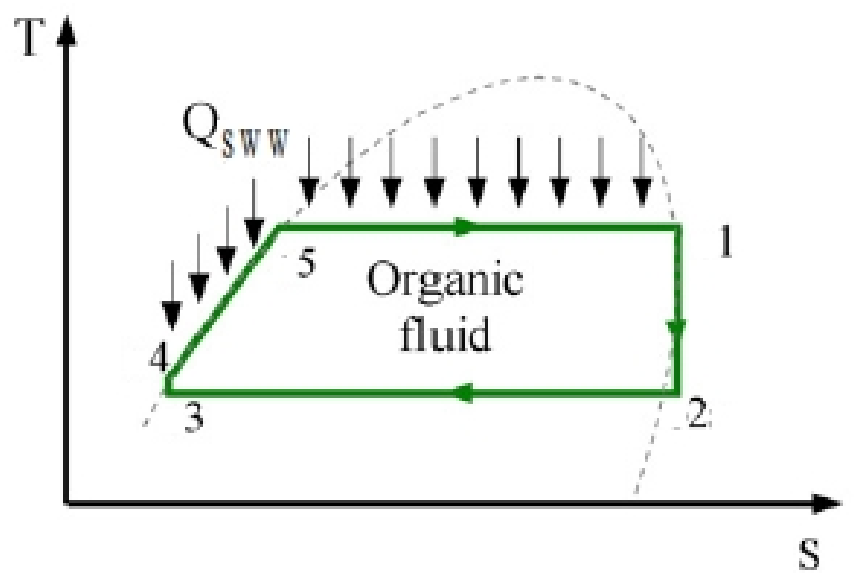

Fig. 2. T-s process diagram of the organic Rankine cycle

This quantity is useful in determining the extent of utilization of the energy contained in the slag washing water, $\phi$ is the quotient of heat flux actually used in the power plant to the amount of available heat flux:

$$
\phi=\frac{Q_{S W W}{ }^{O R C}}{Q_{S W W}}
$$

Where $Q_{S W W}{ }^{O R C}$ is the slag washing water heat flux utilized in the power plant, and it can be expressed as:

$$
Q_{S W W}{ }^{O R C}=m_{S W W} C_{P-S W W}\left(T_{S W W 1}-T_{S W W 2}\right)
$$

Then from the energy balance for the preheater, the injected slag washing water temperature $T_{S W W 2}$ can be determined:

$$
T_{S W W 2}=T_{S W W 3}-\frac{m_{O R C}\left(h_{5}-h_{4}\right)}{m_{S W W} C_{P-S W W}}
$$

According to the scheme shown in Figs. 1 and 2, the working fluid flux $m_{O R C}$ can be determined from the energy balance for the vaporizer: 


$$
\begin{aligned}
& m_{\text {ORC }}=\frac{m_{S W W} C_{P-S W W} \Delta T_{v a p}}{h_{1}-h_{5}} \\
& \Delta T_{v a p}=T_{S W W 1}-T_{S W W 3}
\end{aligned}
$$

For every working fluid, depending on its thermodynamic properties, the optimal value of the temperature drop of the slag washing water in the vaporizer $\left(\Delta T_{v a p}\right)$ that gives the maximum power can be determined [9]. Here in order to simplify the analysis the same temperature drop $\Delta T_{v a p}=20^{\circ} \mathrm{C}$ was assumed for all working fluids. The thermal efficiency and theoretical power of ORC power plant can be calculated as:

$$
\begin{aligned}
& \eta_{t h}=\frac{\left(h_{1}-h_{2}\right)-\left(h_{4}-h_{3}\right)}{h_{1}-h_{4}} \\
& N=m_{\text {ORC }}\left[\left(h_{1}-h_{2}\right)-\left(h_{4}-h_{3}\right)\right]
\end{aligned}
$$

\section{Results}

The temperature of the slag washing water pumped back into the reservoir for the different work fluids are presented in Fig. 3(a). This figure shows that use of an ORC power plant is associated with rather high injection temperatures $T_{S W W 2}$, which are affected by the nature of the organic fluid used in the ORC plant, but to a small extent. In these three work fluids, the injection temperature of R245fa is the highest, and R125 is the lowest. The temperature of slag washing water pumped back into the reservoir has a direct impact on the extent of utilization of this water in the power plant. As can be seen from Fig. 3(b), the different work fluids can use at least $50 \%$ of the available heat flux. With the temperature of initial slag washing water increasing, the available heat flux decreases. The available heat flux of R125 is the highest, and the value of the minimum can reach $70 \%$.
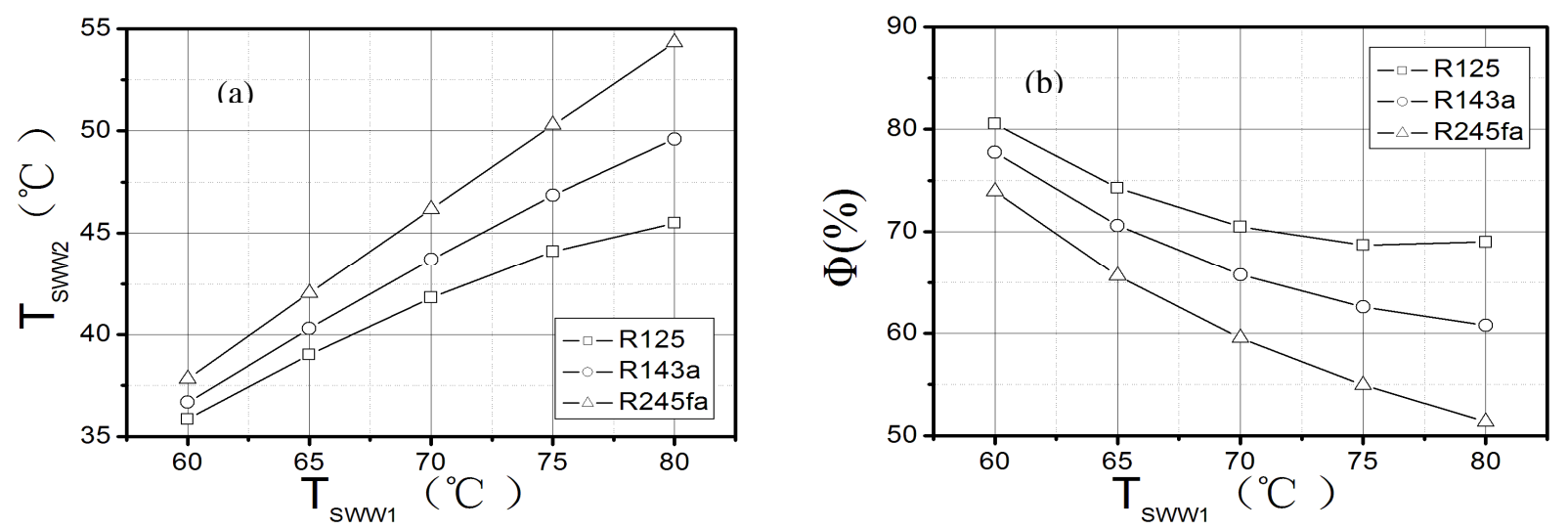

Fig. 3. (a) Temperature of injected slag washing water as a function of $T_{S W W 1}$ for different work fluids.

(b) Utilization of slag washing water energy for the different work fluids as a function of $T_{S W W 1}$

The highest efficiency and thermal power of the ORC of each work fluids depends on the initial temperature of the slag washing water are shown in Fig. 4. According to Fig. 4(a), the highest thermal efficiency is shown by work fluid R245fa, and with the initial slag washing water temperature increasing, the thermal efficiency increases, too. Interestingly, the thermal efficiency of work fluids R125 and R143a are much closed. In Fig.4 (b), the highest theoretical power is shown by work fluid $\mathrm{R} 125$, and with the initial slag washing water temperature increasing, the theoretical power increases, too. Interestingly, when the temperature of initial slag washing water temperature is lower than $70^{\circ} \mathrm{C}$, the work fluids R125 and R245fa have the equal theoretical power. However, when the temperature 
of initial slag washing water temperature is equal into $80^{\circ} \mathrm{C}$, the work fluids R143a and R245fa have the equal theoretical power.
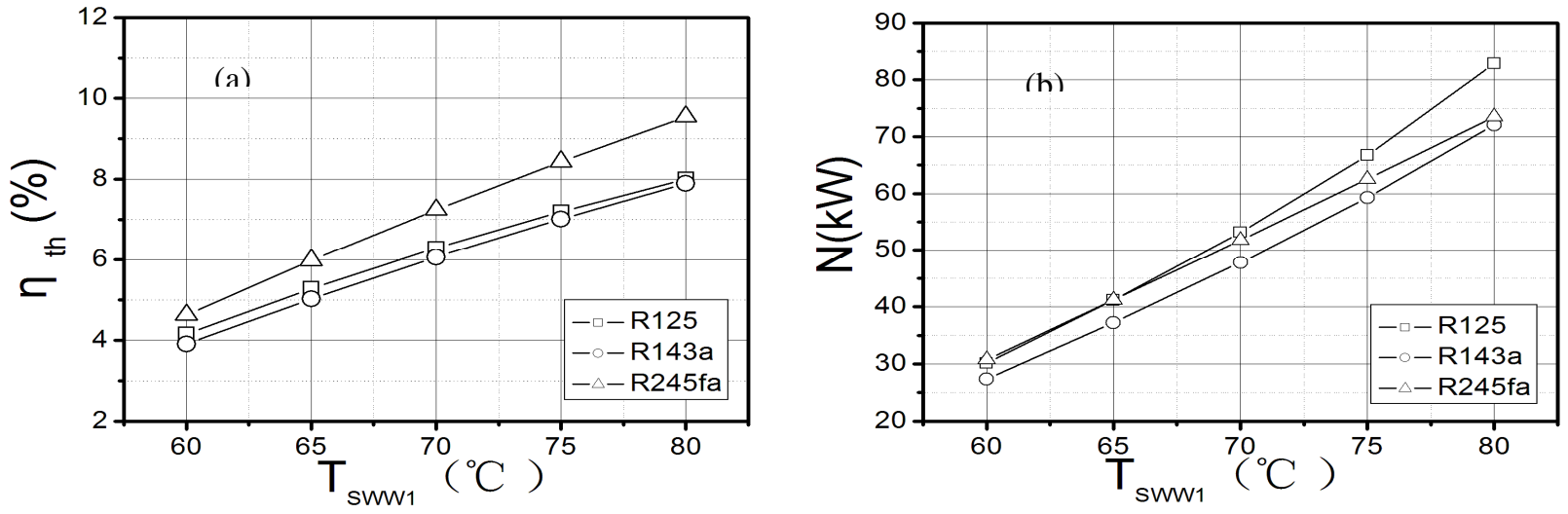

Fig. 4. (a) Thermal efficiency of different work fluids as a function of $T_{S W W 1}$. (b) Theoretical power of different work fluids as a function of $T_{S W W 1}$.

\section{Conclusions}

(1) The different work fluids can use at least $50 \%$ of the available heat flux. With the temperature of initial slag washing water increasing, the available heat flux decreases. The available heat flux of $\mathrm{R} 125$ is the highest, and the value of the minimum can reach $70 \%$.

(2) When the temperature of initial slag washing water temperature is lower than $70^{\circ} \mathrm{C}$, the work fluids R125 and R245fa have the equal theoretical power. However, when the temperature of initial slag washing water temperature is equal into $80^{\circ} \mathrm{C}$, the work fluids R143a and R245fa have the equal theoretical power.

\section{Acknowledgements}

Authors acknowledge the support of Item sponsored by National Natural Science Foundation (Grant No. 51568032 and 21301079).

\section{References}

[1] J.P. Roy, M.K. Mishra and A. Misra: Appl Energ Vol.88 (2011), p. 2995

[2] A. Algieri, P. Morrone: Appl Therm Eng Vol.36 (2012), p. 236

[3] D. Wei, X. Lu and Z. Lu: Applied Thermal Engineering Vol. 28 (2008), p. 1216

[4] J.C. Bruno, J. López-Villada and E. Letelier: Appl Therm Eng Vol. 28 (2008), p. 2212

[5] W. Nowak, A. Borsukiewicz-Gozdur and A. Stache: Applied Energy Vol. 85 (2008), p. 582

[6] H.D.M Hettiarachchi, M.Golubovic and W.M.Worek: Energy Vol. 32 (2007), p. 1698

[7] P.J.Mago, L.M.Chamra and K.Srinivasan: Appl Therm Eng Vol. 28 (2008), p. 998

[8] S. Xiao, S.Y. Wu and D. S. Zheng: J. Cent. South Univ Vol. 20 (2013), p. 737

[9] A. Borsukiewicz-Gozdur, W.Nowak: Appl. Thermal Eng Vol. 27 (2007), p. 2074 